\title{
Evaluation of expression of Toll-Like Receptors 7 and 9, proliferation, and cytoskeletal biomarkers in plaque and guttate psoriasis: A pilot morphological study
}

\author{
Francesca Prignano, ${ }^{1}$ Giulia Lombardo, ${ }^{2}$ Serena Indino, ${ }^{2}$ Federica Ricceri, ${ }^{1}$ Laura Cornaghi, ${ }^{2}$ Elena B. Donetti ${ }^{2}$ \\ ${ }^{1}$ Department of Health Sciences, Section of Dermatology, University of Florence \\ ${ }^{2}$ Department of Biomedical Sciences for Health, University of Milan, Italy
}

\begin{abstract}
This pilot study was aimed at comparing TLR7/TLR9 expression, cytoskeletal arrangement, and cell proliferation by indirect immunofluorescence in parallel lesional and non lesional skin samples of guttate psoriasis (PG) and psoriasis vulgaris (PV) in five male patients for each group $(\mathrm{n}=10)$. TLR7 expression was detected throughout all the epidermal compartment in PV samples, while in PG skin was restricted to the granular layer. TLR9 was present in the granular layer of non lesional skin and in the suprabasal layers of PV/PG lesional skin. Cell proliferation was localized in all the epidermal layers in lesional PG and PV, consistently with the immunopositivity for the "psoriatic keratin" K16. In the suprabasal layers of lesional PG and PV skin, a similar K17 expression was detected and K10 exhibited a patchy distribution. The present results suggest that TLR7 expression can be considered an intrinsic and differential histomorphological feature of PV.
\end{abstract}

Key words: Keratinocyte proliferation; adaptive immunity; keratins; immunofluorescence.

Correspondence: Prof. Elena Donetti, Dipartimento di Scienze Biomediche per la Salute, Università degli Studi di Milano, Via Mangiagalli 31, 20133 Milano, Italy.

Tel. +39.02.50315400 - Fax: +39.02.50315387.

E-mail: elena.donetti@unimi.it

Contributions: FP, FR, ED, designed the research study and contributed to essential reagents, drafted and critically revised the manuscript, dealing with literature search; LC, GL, SI, performed the research and collected the data; FP, ED, analysed the data and developed the interpretation. All the authors have read and approved the final version of the manuscript and agreed to be accountable for all aspects of the work.

Conflict of interest: The authors declare that they have no competing interests, and all authors confirm accuracy.

Availability of data and materials:

Ethical Approval: Patients were selected on consecutive basis to minimize any selection bias after informed written consent and approval by the local Medical Ethical Committee were obtained. 


\section{Introduction}

Guttate or eruptive psoriasis (PG) shares genetic similarities with psoriasis vulgaris (PV), the most represented clinical form. ${ }^{1}$ Clinically, PG is characterized by the eruptive onset of small red papules, followed or preceded by an acute infection such as streptococcal pharyngitis. Scales are not usually clinically visible. In PV, plaque size is highly variable, with erhithematous plaques, covered by silvery white scales, sharply demarcated and, usually, symmetrically distributed. ${ }^{2}$

Epidermal cytotypes and molecules of both the innate and adaptive immunosystem are involved in the pathogenesis and the progression of the disease. ${ }^{3}$ After triggering signals, keratinocytes are able to produce antimicrobial peptides as cathelicidin (LL-37), defensins, and S100 proteins. ${ }^{4,5}$ They do also behave as secretive cells, because they can produce high levels of chemokine CCL20, responsible for further recruiting T-cells into the skin. ${ }^{6}$ Finally yet importantly, keratinocytes express Toll-like Receptors (TLRs), allowing to specifically respond against environmental microbial challenges. In humans, TLR1, TLR2, TLR4, and TLR6 are expressed on the cell surface, whilst TLR3, TLR7, TLR8, and TLR9 are expressed in the cellular components. ${ }^{7}$ TLRs permit to sense endogenous danger associated molecular patterns (DAMPs) and pathogen associated molecular patterns (PAMPs). Many pieces of evidence in literature support the importance of TLR-mediated inflammation in the psoriatic environment. ${ }^{8-11}$

During keratinocyte terminal differentiation, keratin (K) 5 and 14 are typically expressed in the proliferative epidermal compartment, while $\mathrm{K} 1 / \mathrm{K} 10$ in the suprabasal differentiating layers. Specific keratins have been recognized as regulators of many cell functions and as mediators of the epidermal integrity maintenance after a stress. ${ }^{12} \mathrm{~K} 16$ and K17 were indicated as "psoriatic keratins" in the differentiated epidermal layers as they are associated with epidermal hyperproliferation. ${ }^{13}$

The aim of the present study was to compare the morphological features of the psoriatic plaques in PG and PV analysing in parallel lesional and non lesional skin samples and considering cell proliferation, cytoskeletal arrangement, and TLR-mediated innate immunity.

\section{Materials and Methods}

\section{Psoriatic skin collection and quantitative analysis of epidermal proliferation}

Skin punch biopsies $\left(2 \mathrm{~mm}^{2}\right)$ were taken from the gluteal lesional area of five male patients with PV and a Psoriasis Area and Severity Index (PASI) $>10 \%$, and five male patients with PG. Patients were selected on consecutive basis to minimize any selection bias after informed written consent and approval by the local Medical Ethical Committee. All male patients were recruited at Psoriasis Center, Dermatology Unit, University of Florence. A similar punch biopsy was collected in a non lesional area of each patient.

Immediately after surgery, all bioptic samples were incubated for $3 \mathrm{~h}$ at $37^{\circ} \mathrm{C}, 5 \% \mathrm{CO}_{2}$, in low-glucose Dulbecco's modified Eagle's culture medium (Euroclone SpA, Pero, MI, Italy) supplemented with fetal bovine serum (Invitrogen, Carlsbad, CA, USA), penicillin/streptomycin, amphotericin B, glutamine (SigmaAldrich, St. Louis, MO, USA), and $400 \mu \mathrm{mol}$ 5-bromo-2'-deoxyuridine (BrdU), a nonradioactive analogue of thymidine selectively incorporated into the DNA of S-phase cells. Samples were then formalin-fixed, paraffin-embedded, and finally cut by a rotatory microtome.

Briefly, BrdU incorporation was revealed using the mouse antihuman anti-BrdU [Santa Cruz Biotechnology, Santa Cruz, CA, USA; dilution 1:200; $1 \mathrm{~h}$ at room temperature (RT)] after DNA denaturation with $2 \mathrm{~N} \mathrm{HCl}$ (30 min at RT), buffering with $\mathrm{Na}_{2} \mathrm{~B}_{4} \mathrm{O}_{7}(10 \mathrm{~min}$ at RT), saturation of nonspecific binding sites with a solution of $3 \%$ bovine serum albumin (BSA) (Sigma-Aldrich) in PBS 0.1M pH 7.4 (30 min at RT) and digestion with pepsin $0.05 \%$ in $20 \mathrm{mM} \mathrm{HCl} \mathrm{(25} \mathrm{min} \mathrm{at}$ $37^{\circ} \mathrm{C}$ ). Alexa Fluor 488 goat anti-mouse IgG (Thermo Fischer Scientific, Waltham, MA, USA; dilution 1:200; $1 \mathrm{~h}$ at RT) was used as the secondary antibody. Both primary and secondary antibodies were diluted in PBS/BSA 3\%. For the quantitative analysis of keratinocyte proliferation, at least 3 immunofluorescence experiments were carried out for each sample, with 2 slides per sample and 2 sections on each slide. Two independent double-blinded investigators counted the BrdU-positive cells. The results were expressed as the mean number of BrdU-positive cells per square millimeter of living epithelium $\pm 1 \mathrm{SD}$. The statistically significant differences were obtained via Kruskal-Wallis analysis of variance, followed by Dunn's post hoc test.

\section{Immunofluorescence analysis}

Antigen retrieval was always performed incubating slides in $0.01 \mathrm{M}$ citrate buffer $\mathrm{pH} 6$ in microwave, except for TLR9 (in autoclave) and for TLR7 (0.05 M Tris $\mathrm{HCl} \mathrm{pH} 8$ in autoclave). After unspecific binding site saturation with $10 \%$ normal goat serum (Vector Laboratories) in $0.1 \mathrm{M} \mathrm{PBS}(\mathrm{pH} 7.4 ; 30 \mathrm{~min}$ at RT), the following primary antibodies were used: rabbit anti-human TLR7 (Novus Biologicals, Centennial, Co, USA; dilution 1:300 in $0.1 \mathrm{M}$ PBS/BSA 2\%; overnight at $4^{\circ} \mathrm{C}$ ), mouse anti-human TLR9 (Novus Biologicals; dilution 1:10 in 0.1 M PBS; overnight at $4^{\circ} \mathrm{C}$ ), rabbit anti-human K17 (Abcam, Cambridge, UK; dilution 1:200 in 0.1 M PBS/BSA 1\%; overnight at $4^{\circ} \mathrm{C}$ ), mouse anti-human K10 (Santa Cruz Biotechnology; dilution $1: 50$ in $0.1 \mathrm{M}$ PBS/BSA $1 \%$; overnight at $4^{\circ} \mathrm{C}$ ), and rabbit anti-human K16 (Bio SB, Santa Barbara, CA, USA; dilution 1:100 in $0.1 \mathrm{M}$ PBS/BSA $1 \%$; $1 \mathrm{~h}$ at $37^{\circ} \mathrm{C}$ ). Alexa Fluor 488 goat anti-rabbit IgG, Alexa Fluor 488 goat anti-mouse IgG (Thermo Fischer Scientific; dilution 1:200 in 0.1 M PBS/BSA 1\%; $1 \mathrm{~h}$ at RT) or TRITC-conjugated goat anti-rabbit IgG (Jackson ImmunoResearch, West Grove, PA, USA; dilution $1: 200$ in $0.1 \mathrm{M} \mathrm{PBS} / \mathrm{BSA} 1 \%$; $1 \mathrm{~h}$ at RT) were the secondary antibodies. A technical negative control was always considered, omitting the primary antibody. Nuclei were counterstained with 4',6-diamidino-2-phenylindole dihydrochloride (DAPI; Santa Cruz Biotechnology). Immunofluorescence analysis were performed by either Nikon Eclipse E600 wide-field microscope equipped with a Nikon digital camera DXM1200 or Nikon A1R laser scanning confocal microscope.

\section{Results}

\section{Quantitative analysis of epidermal proliferation and K16 expression}

Keratinocyte proliferation was similar in PV and PG non lesional epidermis (Figure 1 a,c). As expected, in all psoriatic lesional samples, a statistically significant increase in proliferation was observed, compared to the respective non lesional samples (Figure 1 b,d). Epidermal proliferation rate in PG samples was the highest among all the considered groups (Figure 1i). K16 positive cells were confined to the basal layer of PV and PG non lesional skin (Figure $1 \mathrm{e}, \mathrm{g}$ ). In lesional samples, K16 immunoreactivity extended from the basal layer upwards both in PV (Figure 1f) and in PG (Figure 1h) lesional skin. 


\section{K10 and K17 immunofluorescence}

K10 expression was homogeneously distributed in the suprabasal layers in non lesional skin of PV and PG (Figure $2 \mathrm{~b}, \mathrm{j}$ ). In $\mathrm{PV}$ and $\mathrm{PG}$ lesional skin, the immunoreactivity was discontinuous and patchy (Figure $2 \mathrm{f,n}$ ).

In non lesional PV and PG samples, K17 was mostly confined in the basal layer (Figure $2 \mathrm{c}, \mathrm{k}$ ), with a very faint immunoreactivity. Conversely, an extensive and intense K17 positivity was found in the spinous and granular layers, with a comparable distribution pattern, in PV and PG lesional skin. The basal compartment exhibited a discontinuous immunoreactivity (Figure 2 g,o). Only scattered keratinocytes in the uppermost epidermal layers showed a co-localization for $\mathrm{K} 10$ and $\mathrm{K} 17$ in PV and PG lesional skin (Figure 2 h,p).

\section{TLR7 and TLR9 expression}

In the granular layer of non lesional PV epidermis, TLR7 expression was always more evident than in the corresponding psoriatic sample (Figure 3a, white arrows). In the basal layer of PV lesional skin, TLR7 immunostaining was more marked than in the respective non lesional samples (Figure $3 b$ ). Conversely, regarding PG, in both non lesional and lesional skin TLR7 immunostaining was similar and confined to the granular layer (Figure $3 \mathrm{c}, \mathrm{d}$ ). TLR9 immunoreactivity was limited to the granular layer in PV and PG non lesional skin (Figure $3 \mathrm{e}, \mathrm{g}$ ). On the other hand, in PV and PG lesional samples TLR9-positive cells were found in the uppermost spinous layer, with only a faint staining in the granular layer (Figure $3 \mathrm{f}$,h; Table 1).

\section{Discussion}

This pilot study describes the main intrinsic features of terminal differentiation, keratinocyte proliferation, and TLR7/TLR9 expression of PG and PV. On the basis of the present observations and according to the existing evidence in literature, we suggest a specific involvement of TLR7 in PV, but not in PG.

Keratinocytes from lesional psoriatic skin overexpressed both TLR7 and TLR9,${ }^{8,9}$ but no morphological data in PV and PG plaque lesions exist. Moreover, no morphological evidence focusing on the epidermal distribution of the acid nucleic sensing TLR7 has been reported in psoriasis. Only recently, RT-PCR investigations described its upregulation in plaques $^{15}$ and peripheral blood mononuclear cells (PBMCs) ${ }^{16}$ in PV patients, revealing a higher TLR7 expression in skin samples than in the respective PBMCs of the same patient. Moreover, the intracellular receptors TLR7 and TLR9 were upregulated in psoriatic PBMCs, independently of PASI. ${ }^{17}$ The distribution pattern of TLR9 is in accordance with the existing literature data, reporting an overexpression of TLR9 in psoriatic lesional skin ${ }^{10}$ and suggesting that the prolonged exposure to an inflammatory milieu, as occuring in PG and PV, induces the TLR9 expression to a variable extent.

A comparison between lesions in PV and PG patients was carried out regarding the expression of TLR2 and TLR4, i.e. the surface TLRs, and highlighted that TLR4 can be related unambiguousely to the pathogenesis of PG. ${ }^{18}$ The results of this pilot study stand in continuation with and complete previous studies, confirming that PV and PG possess both common and intrinsic pathogenetic processes leading to similar, but not identical, clinical outcomes.

No striking differences were detected between PG and PV lesional skin for $\mathrm{K} 10$ and $\mathrm{K} 16 / \mathrm{K} 17$, i.e. the normal and the psoriatic keratins. ${ }^{19,20}$ Consequently, we suggest that K16/K17 induction is a common feature of all psoriatic plaques, in accordance with the existence of a K17/Tcell/cytokine autoimmune loop, ${ }^{21}$ in which K17 represents a multifunctional protein able to regulate also cell proliferation. $^{22}$
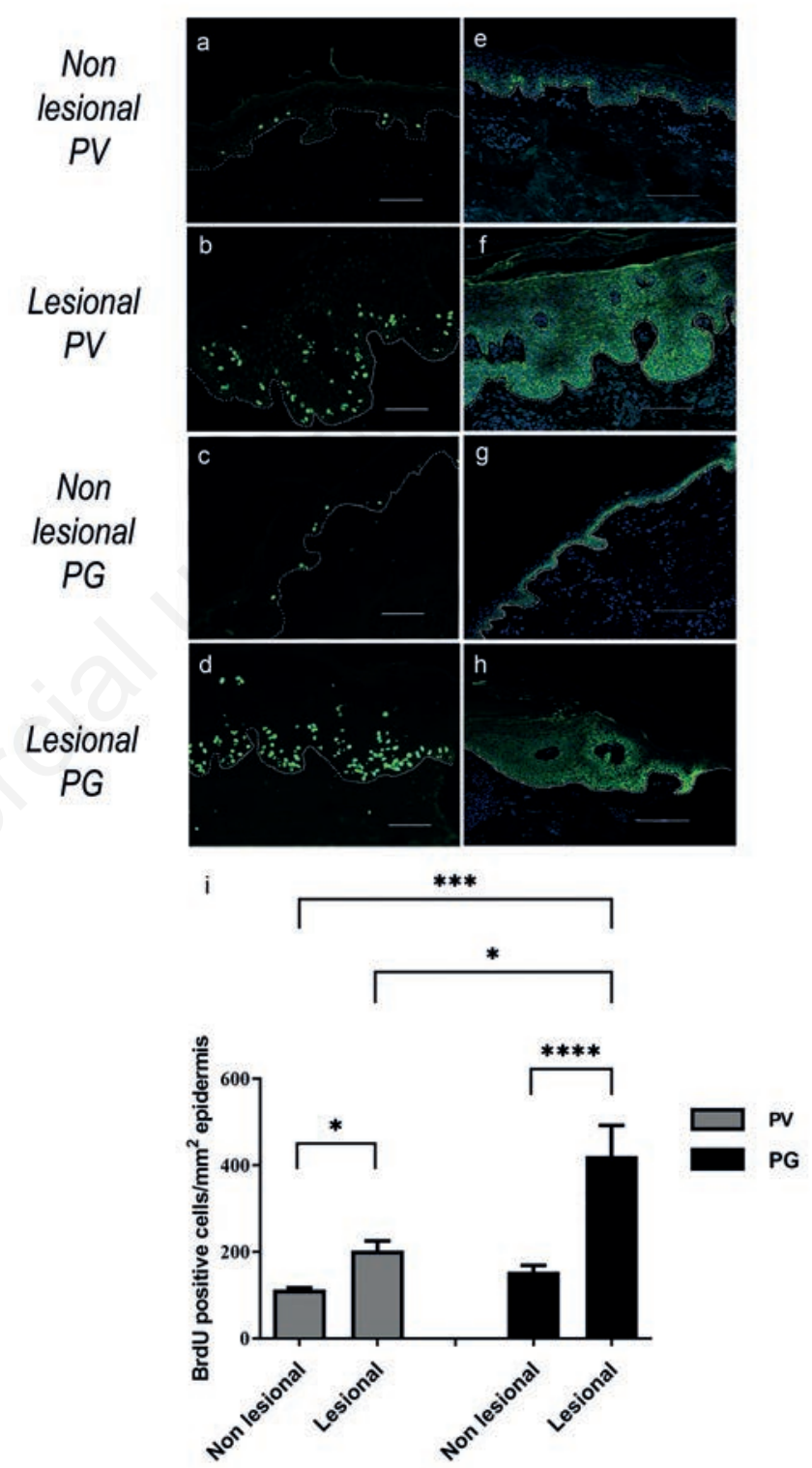

Figure 1. Keratinocyte proliferation and K16 immunostaining in paraffin skin sections. Representative BrdU (a-d) and K16 (e-h) immunostainings, and quantitative analysis of keratinocyte proliferation (i) in non lesional and lesional skin of PV and PG. a,e) Non lesional PV; b,f) lesional PV; c,g): non lesional PG; d,h) lesional PG. Results for quantitative analysis of keratinocyte proliferation are expressed as mean number of BrdU-positive cells per $\mathrm{mm}^{2}$ of living epidermis $+1 \mathrm{SD}$. Statistical analysis was performed via Kruskal-Wallis test followed by Dunn's post hoc test $\left({ }^{*} \mathbf{p}<\mathbf{0 . 0 5},{ }^{* * *} \mathbf{p}<\mathbf{0 . 0 0 1},{ }^{* * * *} \mathbf{p}<\mathbf{0 . 0 0 0 1}\right)$. BrdU, 5-bromo-2'deoxyuridine; K16, keratin 16; PV, psoriasis vulgaris; PG, guttate psoriasis. White dotted line indicates the basal membrane. Scale bars: $100 \mu \mathrm{m}$. 


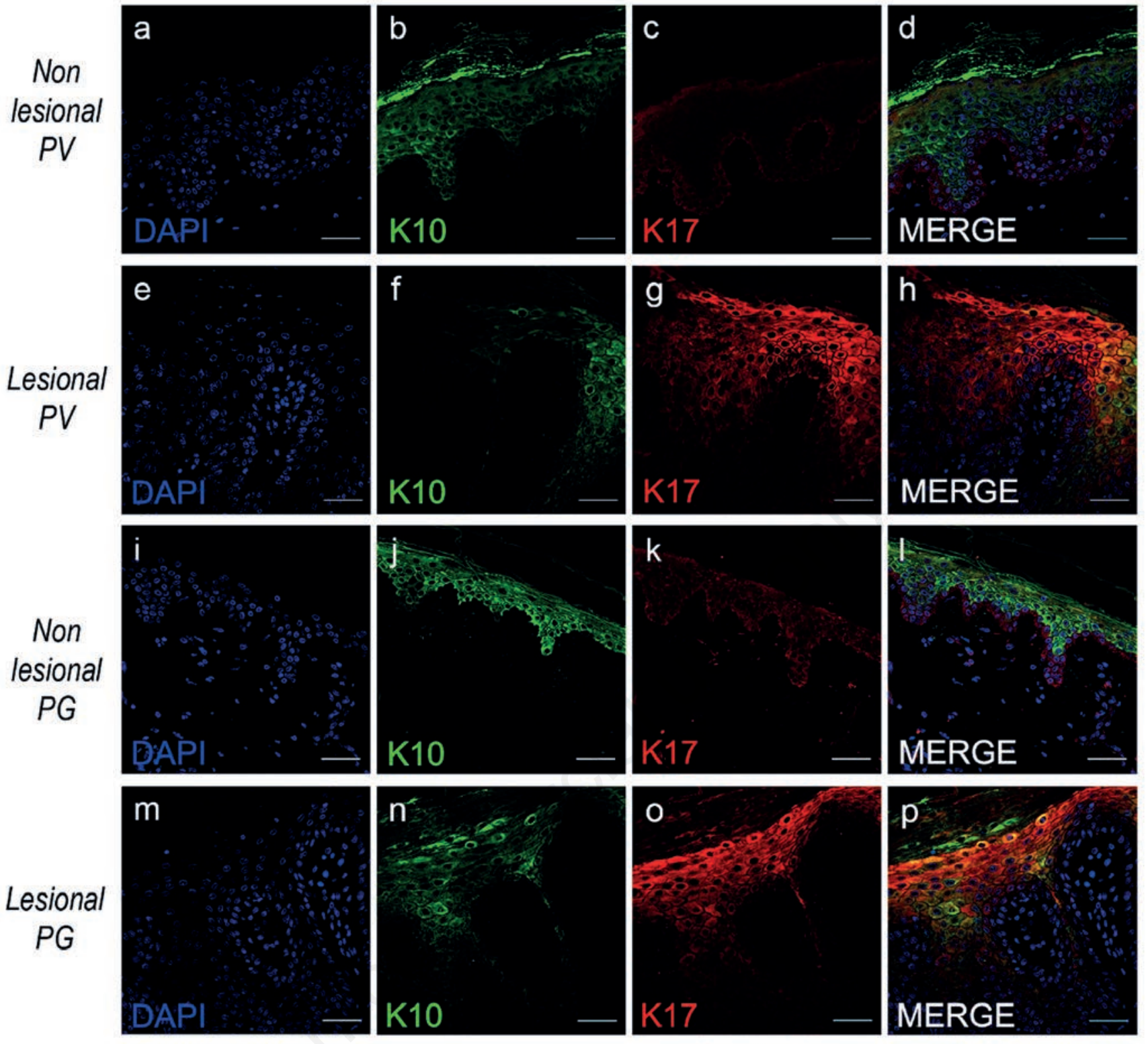

Figure 2. K10/K17 immunostaining in paraffin skin sections. Representative DAPI (a, e, i, m), K10 (b, f, j, n), K17 (c, g, k, o), and K10/K17 (d, h, l, p) immunostainings in non lesional and lesional skin of PV and PG. a-d) Non lesional PV; e-h) lesional PV; i-l) non lesional PG; m-p) lesional PG. DAPI, 4',6-diamidino-2-phenylindole dihydrochloride; K10, keratin 10; K17, keratin 17; PV, psoriasis vulgaris; PG, guttate psoriasis. Scale bars: $50 \mu \mathrm{m}$.

Table 1. Semiquantitative analysis of Toll-like Receptor 7 and 9 in PG and PV non lesional/lesional skin.

\begin{tabular}{|c|c|c|c|c|c|}
\hline & Sample & Basal layer & Lower spinous layer & Upper spinous layer & Granular layer \\
\hline \multicolumn{6}{|c|}{ TLR7 } \\
\hline PV & $\begin{array}{c}\text { Non lesional } \\
\text { Lesional }\end{array}$ & $\begin{array}{l}- \\
+\end{array}$ & $\begin{array}{l}+ \\
+\end{array}$ & $\begin{array}{c}++ \\
+\end{array}$ & $\begin{array}{c}++ \\
+\end{array}$ \\
\hline PG & $\begin{array}{c}\text { Non lesional } \\
\text { Lesional }\end{array}$ & - & - & - & $\begin{array}{l} \pm \\
\pm\end{array}$ \\
\hline \multicolumn{6}{|c|}{ TLR9 } \\
\hline PV & $\begin{array}{c}\text { Non lesional } \\
\text { Lesional }\end{array}$ & - & - & $\begin{array}{c}- \\
++\end{array}$ & $\begin{array}{c}++ \\
\pm\end{array}$ \\
\hline PG & $\begin{array}{c}\text { Non lesional } \\
\text { Lesional }\end{array}$ & $\begin{array}{l}- \\
-\end{array}$ & $\begin{array}{l}- \\
-\end{array}$ & $\begin{array}{c}- \\
++\end{array}$ & $\begin{array}{l}+ \\
\pm\end{array}$ \\
\hline
\end{tabular}

PG, guttate psoriasis; PV, psoriasis vulgaris. 
To our knowledge, this is the first study reporting a quantitative comparison of cell proliferation in PG and PV and showing a higher proliferation rate in $\mathrm{PG}$ compared to PV lesional skin. An issue that should be further addressed is the negative correlation between TLR7 expression and epidermal proliferation in PV. The relevance of adding further insights in this field is actual since small antagonist molecules for human TLR7/9 are more and more charachterized and developed. ${ }^{23,24}$ Similarly to the role attributed to TLR4 in PG, ${ }^{18}$ we suggest that TLR7 expression can be considered an intrinsic and differential histomorphological feature of PV.

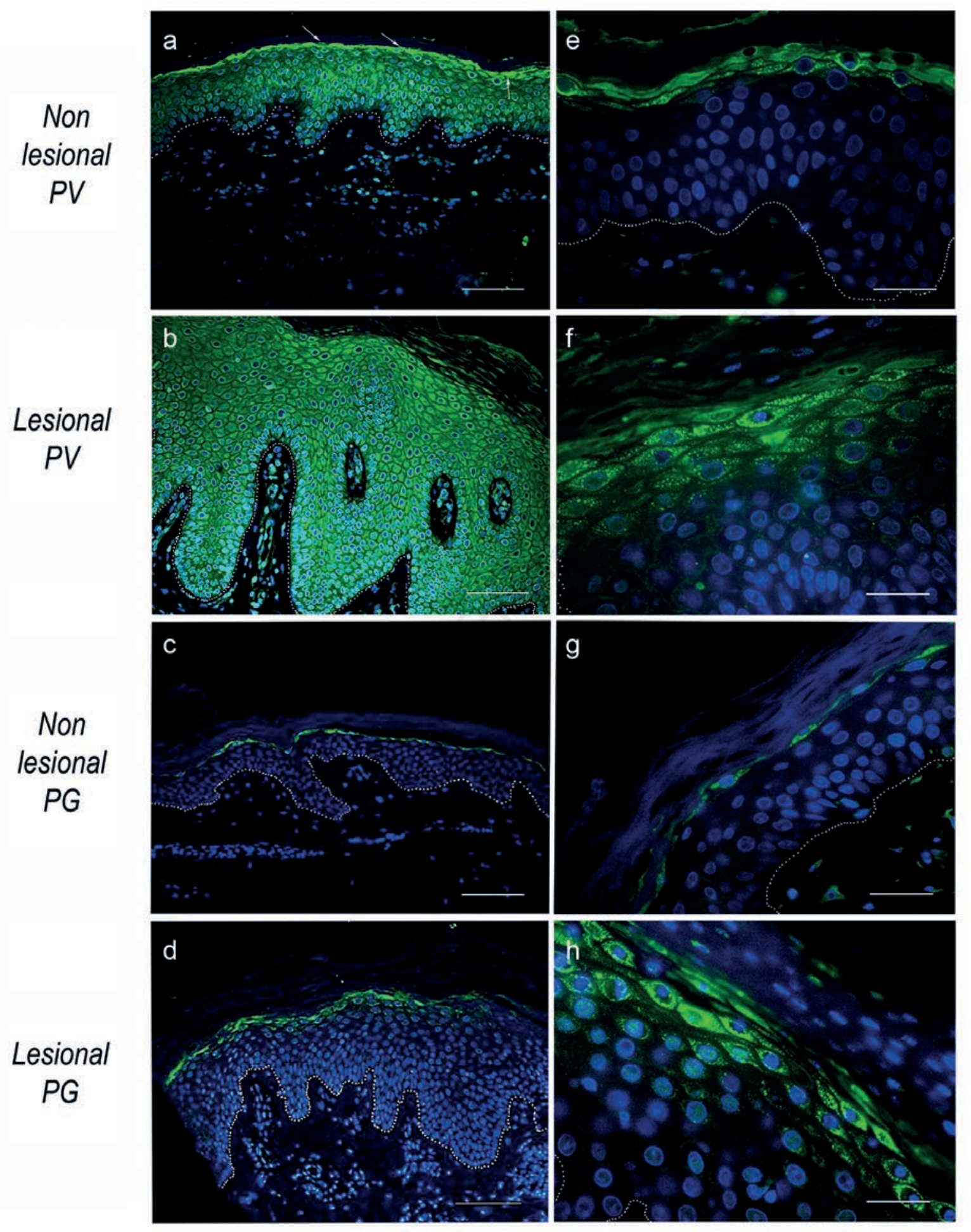

Figure 3. TLR7 and TLR9 immunostaining in paraffin skin sections. Representative TLR7 (a-d) and TLR9 (e-h) immunostainings in non lesional and lesional skin of PV and PG. a,e) Non lesional PV; b,f) lesional PV; c,g) non lesional PG; d,h) lesional PG. TLR7, Tolllike Receptor 7; TLR9, Toll-like Receptor 9; PV, psoriasis vulgaris; PG, guttate psoriasis. White dotted line indicates the basal membrane. Scale bars: a-d) $50 \mu \mathrm{m}$; e-h) $25 \mu \mathrm{m}$. 


\section{Acknowledgements}

We thank the facility Unitech NOLIMITS for the possibility of confocal microscope use and particularly Mrs. Miriam Ascagni for her technical assistance.

\section{References}

1. Nestle FO, Kaplan DH, Barker J. Mechanisms of disease: psoriasis. N Engl J Med 2009;361:496-509.

2. Lebwohl M. Psoriasis. Lancet 2003;361:1197-204.

3. Lowes MA, Suárez-Fariñas M, Krueger JG. Immunology of psoriasis. Annu Rev Immunol 2014;32:227-55.

4. Takahashi T, Gallo RL. The critical and multifunctional roles of antimicrobial peptides in dermatology. Dermatol Clin 2017:35:39-50.

5. Fuentes-Duculan J, Bonifacio KM, Hawkes JE, Kunjravia N, Cueto I, Li X, et al. Autoantigens ADAMTSL5 and LL37 are significantly upregulated in active psoriasis and localized with keratinocytes, dendritic cells and other leukocytes. Exp Dermatol 2017;26:1075-82.

6. Girolomoni G, Mrowietz U, Paul C. Psoriasis: rationale for targeting interleukin-17. Br J Dermatol 2012;167:717-24.

7. Miller LS. Toll-like receptors in skin. Adv Dermatol 2008;24:71-87.

8. Hari A, Flach TL, Shi Y, Mydlarski PR. Toll-like receptors: role in dermatological disease. Mediators Inflamm 2010;2010: 437246.

9. Gilliet M, Conrad C, Geiges M, Cozzio A, Thürlimann W, Burg $\mathrm{G}$, et al. Psoriasis triggered by toll-like receptor 7 agonist imiquimod in the presence of dermal plasmacytoid dendritic cell precursors. Arch Dermatol 2004;140:1490-5.

10. Morizane S, Yamasaki K, Mühleisen B, Kotol PF, Murakami M, Aoyama Y, et al. Cathelicidin antimicrobial peptide LL-37 in psoriasis enables keratinocyte reactivity against TLR9 ligands. J Invest Dermatol 2012;132:135-43

11. Donetti E, Cornaghi L, Arnaboldi F, Ricceri F, Pescitelli L, Maiocchi M, et al. Epidermal barrier reaction to an in vitro psoriatic microenvironment. Exp Cell Res 2017;360:180-8.

12. Karantza V. Keratins in health and cancer: more than mere epithelial cell markers. Oncogene 2011;30:127-38.

13. Donetti E, Lombardo G, Baruffaldi Preis FW, Cornaghi L,
Pescitelli L, Prignano F. 3D skin model to investigate the early epidermal morphological psoriatic features. J Transl Sci 2019;6:1-4.

14. Bedoni M, Sforza C, Dolci C, Donetti E. Proliferation and differentiation biomarkers in normal human breast skin organotypic cultures. J Dermatol Sci 2007;46:139-42.

15. El Tawdy AM, Amin IM, Abdel Hay RM, Hassan AS, Gad, ZS, Rashed LA. Toll-like receptor (TLR)7 expression in mycosis fungoides and psoriasis: a case-control study. Clin Exp Dermatol 2017;42:172-7.

16. Kim HJ, Kim SH, Je JH, Shin DY, Kim DS, Lee MG. Increased expression of Toll-like receptors 3, 7, 8 and 9 in peripheral blood mononuclear cells in patients with psoriasis. Exp Dermatol 2016;25:485-7.

17. Gürel G, Sabah-Özcan S. Evaluation of Toll-like receptor expression profile in patients with psoriasis vulgaris. Gene 2019;702:166-70.

18. Seung NR, Park EJ, Kim CW, Kim KH, Kim KJ, Cho HJ, et al. Comparison of expression of heat-shock protein 60 , Toll-like receptors 2 and 4 , and T-cell receptor $\gamma \delta$ in plaque and guttate psoriasis. J Cutan Pathol 2007;34:903-11.

19. Shi X, Jin L, Dang E, Chang T, Feng Z, Liu Y, et al. IL-17A upregulates keratin 17 expression in keratinocytes through STAT1-and STAT3-dependent mechanisms. J Invest Dermatol 2011;131:2401-8.

20. Zhang W, Dang E, Shi X, Jin L, Feng Z, Hu L, et al. The proinflammatory cytokine il-22 up-regulates keratin 17 expression in keratinocytes via STAT3 and ERK1/2. PLoS One 2012;7:e40797.

21. Shen Z, Chen L, Liu YF, Gao TW, Wang G, Fan XL, et al. Altered keratin 17 peptide ligands inhibit in vitro proliferation of keratinocytes and $\mathrm{T}$ cells isolated from patients with psoriasis. J Am Acad Dermatol 2006;5:992-1002.

22. Yang L, Zhang S, Wang G. Keratin 17 in disease pathogenesis: from cancer to dermatoses. J Pathol 2019;247:158-65.

23. Mussari CP, Dodd DS, Sreekantha RK, Pasunoori L, Wan H, Posy SL, et al. Discovery of potent and orally bioavailable small molecule antagonists of Toll-like receptors 7/8/9 (TLR7/8/9). ACS Med Chem Lett 2020;11:1751-8.

24. Patinote C, Karroum NB, Moarbess G, Cirnat N, Kassab I, Bonnet PA, et al. Agonist and antagonist ligands of toll-like receptors 7 and 8: Ingenious tools for therapeutic purposes. Eur J Med Chem 2020;193:112238.

Received for publication: 11 January 2021. Accepted for publication: 18 February 2021.

This work is licensed under a Creative Commons Attribution-NonCommercial 4.0 International License (CC BY-NC 4.0).

(C) Copyright: the Author(s), 2021

Licensee PAGEPress, Italy

European Journal of Histochemistry 2021; 65:3218

doi:10.4081/ejh.2021.3218 